\title{
CORRECTION
}

\section{Author Correction: Embryonic senescent cells re-enter cell cycle and contribute to tissues after birth}

Yi Li ${ }^{1,2}$, Huan Zhao ${ }^{1,2}$, Xiuzhen Huang ${ }^{1,2}$, Juan Tang ${ }^{1,2}$, Shaohua Zhang ${ }^{1}$, Yan $\mathrm{Li}^{1,2}$, Xiuxiu Liu ${ }^{1}$, Lingjuan He ${ }^{1,2}, \mathrm{Zhenyu} \mathrm{Ju}^{3}$, Kathy O. Lui ${ }^{4}$ and Bin Zhou (iD) $1,2,3,5$

Cell Research (2018) 28:871; https://doi.org/10.1038/s41422-018-0063-1

Correction to: Cell research (2018) 0:1-4. https://doi.org/10.1038/ s41422-018-0050-6; published online 05 June 2018
The authors apologized for the spelling mistake of author name for Zhengyu Ju. The correct form is Zhenyu Ju. We apologize for any hassle that may have been caused by our mistake.

\footnotetext{
${ }^{1}$ State Key Laboratory of Cell Biology, CAS Center for Excellence in Molecular Cell Science, Institute of Biochemistry and Cell Biology, Shanghai Institutes for Biological Sciences, Chinese Academy of Sciences, University of Chinese Academy of Sciences, 200031 Shanghai, China; ${ }^{2}$ Key Laboratory of Nutrition and Metabolism, Institute for Nutritional Sciences, Shanghai Institutes for Biological Sciences, Chinese Academy of Sciences, University of Chinese Academy of Sciences, 200031 Shanghai, China; ${ }^{3}$ Key Laboratory of

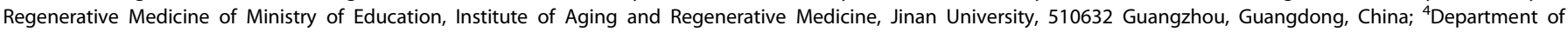
Chemical Pathology, Li Ka Shing Institute of Health Sciences, Prince of Wales Hospital, The Chinese University of Hong Kong, Shatin, Hong Kong SAR, China and ${ }^{5}$ School of Life Science and Technology, ShanghaiTech University, 201210 Shanghai, China

Correspondence: Bin Zhou (zhoubin@sibs.ac.cn)
}

Published online: 29 June 2018 